\title{
On the Topological Characterization of Robot Singularity Loci. A Catastrophe-Theoretic Approach
}

\author{
Federico Thomas and Philippe Wenger
}

\begin{abstract}
Two-dimensional slices of robot singularity loci contain, in general, cusps. This kind of points are important because their presence indicates the possibility of planning assembly-changing motions that do not meet any singularity. The critical points where the number of cusps changes, as the slice is swept, permit one to decompose a singularity locus into domains where the obtained slices share common topological properties. In this paper, it is shown how Catastrophe Theory provides a solid framework to classify these critical points up to certain types of equivalence giving precise local models to describe them. It is also shown how only three possible types of these critical points exist for generic robots. The presented results are exemplified on a serial $3 R$ robot.
\end{abstract}

\section{INTRODUCTION}

It is well known that the inverse kinematics of serial robots and the forward kinematics of parallel robots have, in general, multiple solutions known as assembly modes. When two assembly modes coincide (two solution branches of the kinematics problem meet), the robot is in a singularity. In one of these singularities the robot becomes uncontrollable because, roughly speaking, it is impossible to decide which assembly mode the robot will adopt when getting out of it. Thus, avoiding robot singularities is critical but, to this end, they should be previously characterized. The literature on identification, avoidance and analysis of robot singularities is certainly extensive. The interested reader is addressed to the online database available at [1].

In order to obtain the assembly modes of a particular robot, it is always possible to manipulate its kinematic equations to reduce the problem to finding the roots of a univariate polynomial, known as the characteristic polynomial of the robot. When using this algebraic elimination approach for obtaining the assembly modes, it is advisable to determine the sequence of variable elimination that leads to the polynomial with the lowest possible degree. At the end of this process, one comes up with a polynomial of the form:

$$
f\left(t, \omega_{1}, \ldots, \omega_{n}\right)=\sum_{i=0}^{n} a_{i}\left(\omega_{1}, \ldots, \omega_{n}\right) t^{i}=0,
$$

where the coefficients $a_{i}$ depend on the geometry of the robot - which is constant for each particular robot instance - and on the input variables $\omega_{i}, i=1, \ldots, n$. These input variables are the variables associated with the actuators, when solving

F. Thomas is with the Institut de Robòtica i Informàtica Industrial (CSIC-UPC), Llorens Artigas 4-6, 08028 Barcelona, Spain. E-mail: fthomas@iri.upc.edu. Ph. Wenger is with the Institut de Recherche en Communications et Cybernétique de Nantes (UMR-CNRS), 1, rue la Noe, BP 9210144321 Nantes Cedex 3, France. E-mail: Philippe.Wenger@irccyn.ecnantes.fr. the forward kinematics of parallel robots, or the variables defining the pose of the end effector, when solving the inverse kinematics of serial robots. For a given set of values of these input variables, each real root of the above polynomial in $t$ determines an assembly mode. Since a singularity is reached when two solution branches meet, the singularities correspond to values of $\omega_{i}, i=1, \ldots, n$ for which (1) has a double root. This is certainly an oversimplified view of the problem but this will be enough for the purposes of this paper whose main goal is to draw the attention of the reader to some tools developed in the area of Catastrophe Theory which are of interest in the characterization of robot singularities.

Equation (1) can be seen as the equation of a hypersurface in implicit form in the space defined by $\left(\omega_{1}, \ldots, \omega_{n}, t\right) \in$ $\mathbb{R}^{n+1}$. To get some insight into the nature of this surface, we can start by fixing all but two input variables, say $\omega_{1}$ and $\omega_{2}$. Then, we can define

$$
G\left(t, \omega_{1}, \omega_{2}\right) \doteq f\left(t, \omega_{1}, \omega_{2}, \omega_{3}^{0}, \ldots, \omega_{n}^{0}\right)=0,
$$

where $\omega_{3}^{0}, \ldots, \omega_{n}^{0}$ correspond to the fixed inputs. Then, the singularities of the robot in this subspace are the double roots of (2). In other words, the solutions to the system:

$$
\left.\begin{array}{l}
G\left(t, \omega_{1}, \omega_{2}\right)=0 \\
G_{t}\left(t, \omega_{1}, \omega_{2}\right)=0
\end{array}\right\}
$$

where $G_{t}$ denotes the partial derivative of $G$ with respect to $t$. By eliminating $t$ in the above system, we get the resultant

$$
H\left(\omega_{1}, \omega_{2}\right)=0,
$$

which can be seen as the equation of a curve, in implicit form, resulting from projecting orthographically, onto the plane defined by $\omega_{1}$ and $\omega_{2}$, those points of $G\left(t, \omega_{1}, \omega_{2}\right)=0$ where the direction defined by $t$ is tangent to the surface. These curves are called apparent contours [2] which divide the subspace defined by $\omega_{1}$ and $\omega_{2}$ into regions where the number of assembly modes is constant and, depending on how these regions are distributed, interesting results can already be drawn. For example, it has been shown that it is possible to change from one assembly mode to another one without meeting any singularity at least in two situations: by encircling a cusp [3] or by encircling a so-called $\alpha$-curve [4] (see Fig. 1).

Now, let us suppose that we continuously modify one of the input variables that we have previously left fixed. Then, the resulting apparent contours can be described in terms of smooth changes that do not modify its topology, and a set 

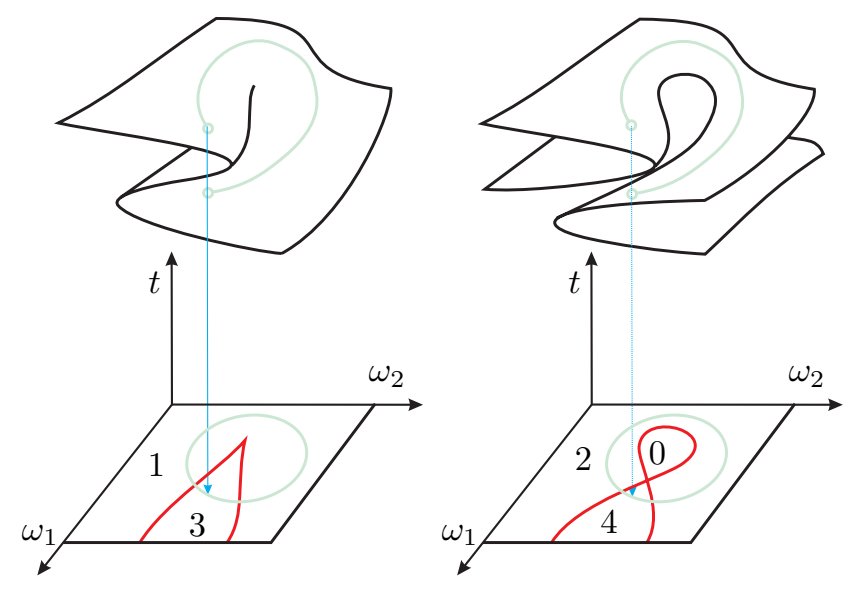

Fig. 1. Encircling a cusp (left) or an $\alpha$-curve (right) allows, under some circumstances, to change the assembly mode of a robot without trespassing a singularity. The numbers appearing on the plane defined by $\omega_{1}$ and $\omega_{2}$ indicate the number of assembly modes.

of critical points that lead to abrupt transitions. A similar situation arises in Computer Vision when characterizing the different topological aspects obtained from monocular views of a 3D object with an algebraic boundary [5], [6].

In what follows, we will assume, without loss of generality, that the apparent contours are obtained by projecting onto the subspace defined by $\omega_{1}$ and $\omega_{2}$ and we want to detect the abrupt changes in the topology of this projection as $\omega_{3}$ is swept in its range, while all other input variables remain fixed. Then, we can define

$$
F\left(t, \omega_{1}, \omega_{2}, \omega_{3}\right) \doteq f\left(t, \omega_{1}, \omega_{2}, \omega_{3}, \omega_{4}^{0}, \ldots, \omega_{n}^{0}\right)=0 .
$$

This paper is devoted to the analysis of the structural stable properties of the orthographic projections of the hypersurface implicitly defined by (5). To this end, Catastrophe Theory and its application to the projection of smooth surfaces (see [7, Chapter 12] for an introduction) is employed. We will see how this theory provides a solid framework to classify critical points up to certain types of equivalence giving precise local models to describe them. The presented results are not new but they are rather scattered in the literature and most of the time presented in an exceedingly difficult language to those unused to it. We make here an effort to present them in an accessible language to a robotics audience.

This paper is structured as follows. Section II presents the different types of singularities arising when projecting a smooth surface. Section III shows how the three types of critical points arising when projecting a generic smooth surface - the lips, beaks and swallowtail singularities- bifurcate into cusps and how they can be identified analytically. Section IV uses a 3R spatial serial robot to exemplify these three bifurcations. Finally, Section V summarizes the main points presented and those that deserve further attention.

\section{Apparent Contours And Singularity Loci}

Following the description of the problem of computing the apparent contours of a smooth surface due to Callahan
[8], take a piece of fabric, crumple it and press it on a flat surface. In general, the sheet can assume three shapes around a given point [see Fig. 2(left)]: (a) a flat and smooth region, (b) a fold line, and (c) a point where a pleat is formed, usually known as a cusp. Some experimentation leads to the conclusion that other cases are possible (point $d$ in Fig. 2(left) is a new type, for instance) but they disappear by any small perturbations in the position of the fabric. For example, the new shape at $d$ in Fig. 2(left) disappears as soon as the the bottom fold is slightly pulled [Fig. 2(right)]. Points of type $a, b$, and $c$ cannot be made to disappear by small perturbations. Their position can be affected, but not its presence. The preceding observations amount to an informal paraphrase proved by Whitney in 1955 [9] in one of the founding papers of Catastrophe Theory.
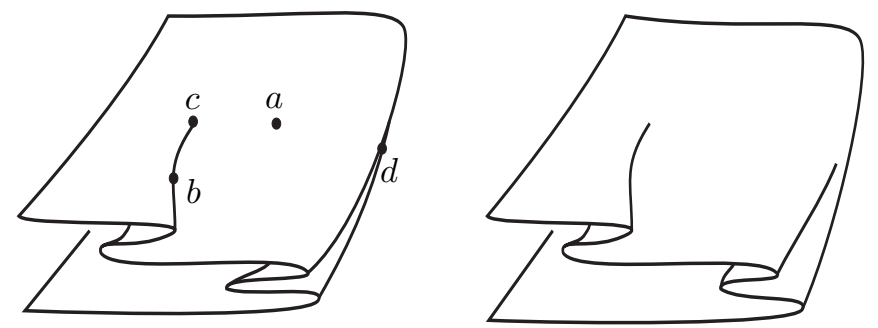

Fig. 2. Contrarily to what happens with points of type $d$, those of type $a$, $b$, and $c$ cannot be made to disappear by small perturbations; their position can be affected, but not its presence. Adapted from [8].

If the projection direction is generic, the apparent contour is composed of cusps and folds. However, if this direction is chosen in a special way, one may also obtain some nonstable singularities. Mathematicians measure degeneracy by codimension, roughly speaking the number of independent conditions that need to be imposed to achieve a degeneracy. Thus, these non-stable singularities are often classified according to their codimension. Cusps and folds are referred to as being of codimension 0 . Singularities of codimension greater than 0 partition the space of all possible projections into aspects, separated by bifurcation sets. Describing all possible non-stable singularities —or critical points, as we call them in the previous section - and how they bifurcate into stable singularities can be considered straightforward but it is certainly an error-prone difficult task solved by several mathematicians at the beginning of the eighties. The names of Platonova, Shcherbak, Arnold, McCrory, Rieger, and Kergosien will remain associated with the consecution of this classification [10], [11], [12], [7]. Next, we give a concise presentation of its derivation.

It is said that a tangent vector in some point has contact of order $n$ with a surface when de directional derivatives of order $i$ of the surface equation in the direction of the tangent are zero for all $i<n$, and nonzero for $i=n$. All surface points have an infinity of tangents of order 2 in their tangent plane. By definition, a tangent of order 3 or greater is called an asymptotic tangent, and its direction at the point of tangency an asymptotic direction. Elliptic points do not have asymptotic tangents, parabolic points have one, and 
TABLE I

THE 9 SINGULARITIES OF CODIMENSION 0, 1, AND 2 (THE BUTTERFLY SINGULARITY ENCOMPASSES TWO GERMS)

\begin{tabular}{|c|c|c|c|c|}
\hline Codimension & Germ & Name & Geometry & Description \\
\hline \multirow[t]{2}{*}{0} & $z=x^{2}$ & Fold & & $\begin{array}{l}\text { The } t \text {-axis is parallel to a tangent of order } 1 \text {. The tangency } \\
\text { occurs at a point in an elliptic domain. }\end{array}$ \\
\hline & $z=x^{3}+x y$ & Cusp & & $\begin{array}{l}\text { The } t \text {-axis is parallel to a tangent or order } 2 \text {. The tangency } \\
\text { occurs at a point in a hyperbolic domain. }\end{array}$ \\
\hline \multirow[t]{3}{*}{1} & $z=x^{3}+x y^{2}$ & Lips & 0 & $\begin{array}{l}\text { The } t \text {-axis is parallel to a tangent of order } 2 \text {. The tangency } \\
\text { occurs at a point of a parabolic curve. Two cusps sharing both } \\
\text { branches are created or destroyed when passing through this } \\
\text { singularity. }\end{array}$ \\
\hline & $z=x^{3}-x y^{2}$ & Beaks & & $\begin{array}{l}\text { The } t \text {-axis is parallel to a tangent of order } 2 \text {. The tangency } \\
\text { occurs at a point of a parabolic curve. Two cusps sharing } \\
\text { neither branch are created or destroyed when passing through } \\
\text { this singularity. }\end{array}$ \\
\hline & $z=x^{4}+x y$ & Swallowtail & & $\begin{array}{l}\text { The } t \text {-axis is parallel to a tangent of order } 3 \text {. The tangency } \\
\text { occurs at a point of a flecnodal curve. Two cusps sharing a } \\
\text { branch are created or destroyed. }\end{array}$ \\
\hline \multirow[t]{3}{*}{2} & $z=x^{3}+x y^{3}$ & Goose & & $\begin{array}{l}\text { The tangency occurs at a point of a parabolic curve. Transition } \\
\text { between lips and beaks. }\end{array}$ \\
\hline & $z=x^{4}+x^{2} y+x y^{2}$ & Gulls & & $\begin{array}{l}\text { The tangency occurs at a point of a flecnodal curve where the } \\
\text { t-axis is parallel to a tangent of order } 4 \text {. The tangency occurs } \\
\text { at a godron. Transition between beaks and swallowtail. }\end{array}$ \\
\hline & $z=x^{5}+x^{3} y \pm x y$ & Butterfly & & $\begin{array}{l}\text { The tangency occurs at a point of a flecnodal curve where } \\
\text { the t-axis is parallel to a tangent of order } 4 \text {. The tangency } \\
\text { occurs at a biflecnode. Transition between two swallowtails } \\
\text { that coalesce sharing a cusp. }\end{array}$ \\
\hline
\end{tabular}

hyperbolic points have two. It can be shown that the line of projection is of order 2 at a fold point and of order 3 at a cusp. The interesting thing arrives when one realizes that higher-order non-stable singularities also correspond to higher order contacts between the line of projection and the surface. Thus the first step is to classify all surface points according to the order of contacts of their tangents. The task gets simplified by realizing that generic surfaces have no tangents of order greater than 5 [7].

Then, it is possible to classify the points of a generic surface into the following seven classes according to the orders of their tangents (Fig. 3) [10]:

(1) Elliptic domain: Open domain of the surface whose points have no asymptotic tangents;

(2) Hyperbolic domain: Open domain of the surface whose points have two asymptotic tangents; and

(3) Parabolic curve: A smooth curve embedded in the surface whose points have a unique, but double, asymptotic tangent. The parabolic curve divides the surface into the elliptic and hyperbolic domains [13].

In the closure of the hyperbolic domain we might also encounter a: 
(4) Flecnodal curve: Set of points at which one of the asymptotic tangents exceeds order three. Moreover, one may also encounter isolated points of the following three types [14]:

(5) Godron: A parabolic point at which the (unique) asymptotic direction is tangent to the flecnodal curve.

(6) Hyperbonode: A point of self-intersection of the flecnodal curve (points with two asymptotic tangents of order higher than three).

(7) Biflecnode: A point of the flecnodal curve at which one asymptotic tangent exceeds order four.

The above seven classes are robust features of a smooth surface, that is, they are stable in the sense that under a sufficiently small perturbation they do not vanish but only deform. It is worth noting that these seven classes were already known at the end of the 19th century in the context of the enumerative theory of complex algebraic surfaces [15].

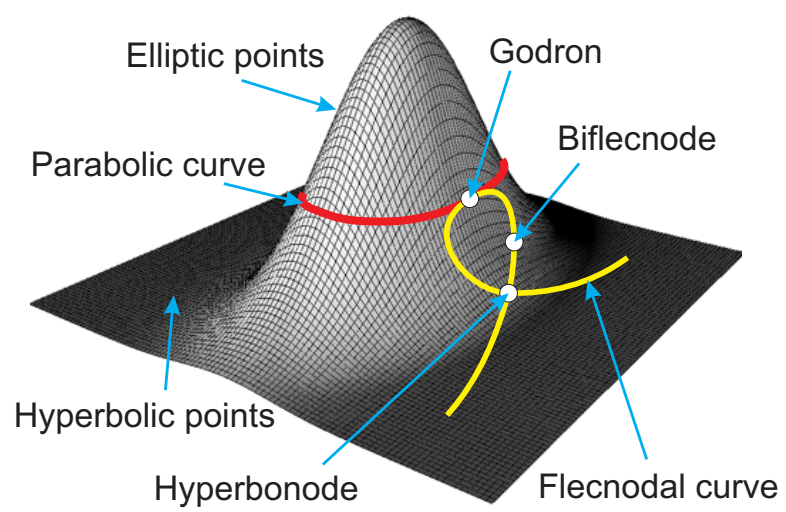

Fig. 3. Classification of the points in a smooth surface according to the order of their tangents.

The classes of points of a generic surface appear in the form of the various singularities of its apparent contours. If the direction of projection is generic, then by Whitney's theorem the only singularities are folds and cusps. However, if one has chosen the direction of projection in a special way, one may also obtain certain non-generic projections of a generic surface. It turns out that all such projections reduce locally to the projection of one of the nine surfaces of the form $z=f(x, y)$ along the $\mathrm{x}$-axis onto the $(y, z)$ plane, technically known as germs, that appear in Table I. In our case, the reduction is obtained through a change of variables of the form $\left(x\left(t, \omega_{1}, \omega_{2}\right), y\left(\omega_{1}, \omega_{2}\right), z\left(\omega_{1}, \omega_{2}\right)\right)$. Each of these germs have received names chosen for a variety of reasons and should be treated merely as mnemonics. Platonova proved that the total number of nonequivalent singularities of projections of generic smooth surfaces from all points in 3D space is 14 [10]. This apparent contradiction with the classification given in Table I is due to the fact that the missing singularities are not obtained from orthographic projections but from central projections at very particular points [16].
On a modification of the surface defined by $F\left(t, \omega_{1}, \omega_{2}, \omega_{3}\right)=0$, as $\omega_{3}$ varies, at isolated moments the singularities of codimension 1 will appear. Singularities of codimension 2 occur only at isolated projecting directions, and one needs to search for them deliberately. The singularities of codimension 2 have interest when considering one more input variable, say $\omega_{4}$. In this case, singularities of codimension 1 become stable and singularities of codimension 2 will appear at isolated points that bifurcate into those of dimension 1 while varying $\omega_{3}$ and $\omega_{4}$. Clearly, when considering only three input variables and generic geometric parameters, singularities of codimension 2 do not need to be considered and we can limit our analysis to singularities up to codimension 1 .

\section{The Three Singularities OF CODIMENSION 1 AND THEIR BIFURCATIONS}
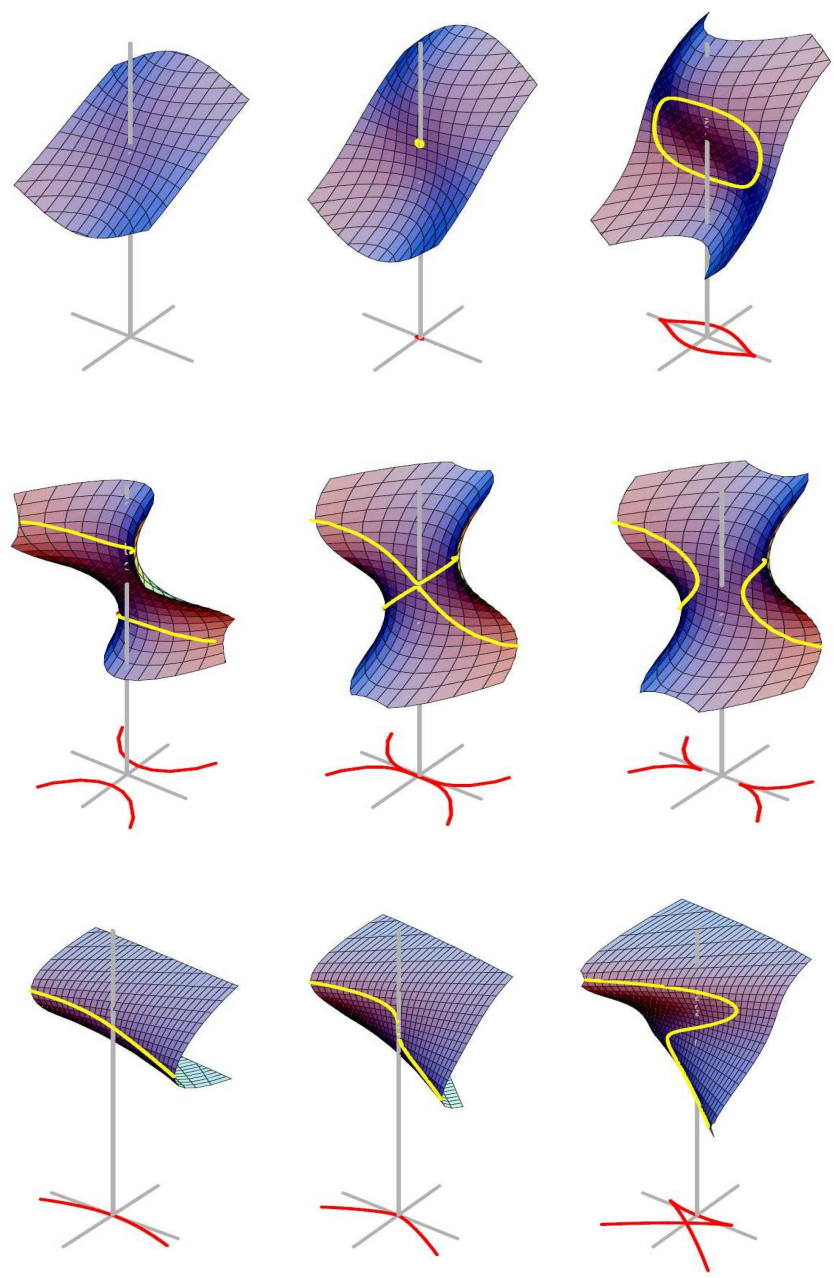

Fig. 4. The three singularities of codimension 1 and their bifurcations. The lips (top), the beaks (center), and the swallowtail (bottom) singularities bifurcate as depicted. Adapted from [17].

The characterization of the singularities of codimension 1 give essential information on the genesis of cusps. Indeed, consider the three surfaces and their apparent contours depicted in the leftmost column of Fig. 4. By properly 
modifying the parameters of these surfaces, a lips, beaks and swallowtail singularities can be obtained, as shown respectively in the center column. By further modifying these parameters, two cusps arise in each of the three cases, as shown in the rightmost column. It is important to realize that cusps always appear in pairs and that, by locating the singularities of codimension 1 , it is possible to find domains of the input variables for which the number of cusps is constant. Next, we give an algebraic characterization of these singularities.

To obtain a beaks or lips singularity, the projection line must have a contact of order 3 with the surface, i.e., the point of contact must be a point of a parabolic curve. This can be expressed by the following system:

$$
\left.\begin{array}{l}
F\left(t, \omega_{1}, \omega_{2}, \omega_{3}\right)=0 \\
F_{t}\left(t, \omega_{1}, \omega_{2}, \omega_{3}\right)=0 \\
F_{t t}\left(t, \omega_{1}, \omega_{2}, \omega_{3}\right)=0 \\
\Phi\left(t, \omega_{1}, \omega_{2}, \omega_{3}\right)=0
\end{array}\right\}
$$

where

$$
\begin{aligned}
\Phi\left(t, \omega_{1}, \omega_{2}, \omega_{3}\right)= & F_{\omega_{1}}^{2}\left(F_{\omega_{2} \omega_{2}} F_{t t}-F_{\omega_{2} t}^{2}\right) \\
& +F_{\omega_{2}}^{2}\left(F_{\omega_{1} \omega_{1}} F_{t t}-F_{\omega_{1} t}^{2}\right) \\
& +F_{t}^{2}\left(F_{\omega_{1} \omega_{1}} F_{\omega_{2} \omega_{2}}-F_{\omega_{1} \omega_{2}}^{2}\right) \\
& +2 F_{\omega_{1}} F_{\omega_{2}}\left(F_{\omega_{1} t} F_{\omega_{2} t}-F_{t t} F_{\omega_{1} \omega_{2}}\right) \\
& +F_{\omega_{2}} F_{t}\left(F_{\omega_{1} \omega_{2}} F_{\omega_{1} t}-F_{\omega_{1} \omega_{1}} F_{\omega_{2} t}\right) \\
& +2 F_{\omega_{1}} F_{t}\left(F_{\omega_{1} \omega_{2}} F_{\omega_{2} t}-F_{\omega_{2} \omega_{2}} F_{\omega_{1} t}\right)=0
\end{aligned}
$$

is the expression in the first and the second partial derivatives of $F\left(t, \omega_{1}, \omega_{2}, \omega_{3}\right)$ with respect to $t, \omega_{1}, \omega_{2}$ that characterizes the parabolic curves parameterized in $\omega_{3}$ [18].

To obtain a swallowtail singularity, the projection line must have a contact of order 4 with the surface; i.e., the point of contact must be a point of a flecnodal curve. This can be expressed with the system

$$
\left.\begin{array}{l}
F\left(t, \omega_{1}, \omega_{2}, \omega_{3}\right)=0 \\
F_{t}\left(t, \omega_{1}, \omega_{2}, \omega_{3}\right)=0 \\
F_{t t}\left(t, \omega_{1}, \omega_{2}, \omega_{3}\right)=0 \\
F_{t t t}\left(t, \omega_{1}, \omega_{2}, \omega_{3}\right)=0
\end{array}\right\}
$$

Solving systems (6) or (7) via resultants is, in general, unfeasible because the resulting polynomial degree is very high even for simple robots. Continuation or interval-based methods seem the only alternatives. Nevertheless, ad hoc simplified algebraic characterizations can be found for some robots. This is the case the orthogonal 3R serial robot, as shown in the next section.

Finally, it is worth to mention that, besides beaks, lips and swallowtail singularities, three more singularities should be taken into account because they introduce changes in the topology of the apparent contours though they do not alter the number of cusps. They are global singularities commonly known as triple points, tangent crossings, and cusp crossings. Their algebraic characterization can be found in [18].

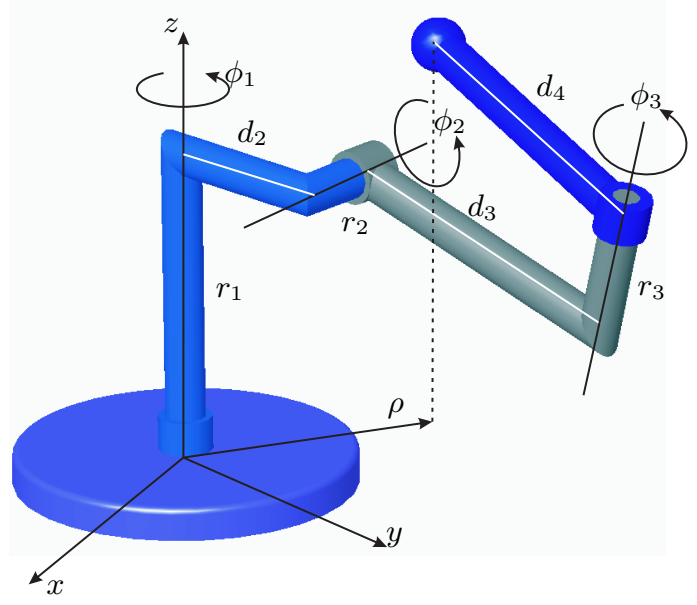

Fig. 5. An orthogonal 3R robot and associated notation.

\section{Example: The orthogonal 3R Serial Robot}

Figure 5 depicts a serial $3 \mathrm{R}$ robot with mutually orthogonal joint axes. This robot was analyzed in detail in [19]. In this case the input variables are the cartesian coordinates of the end effector, $x, y$, and $z$. Due to the symmetry of the inverse kinematics problem with respect to the $z$-axis, the input variables can be chosen to be $\rho=\sqrt{x^{2}+y^{2}}$ and $z$. The singularity curves in the plane defined by these two variables are determined by a polynomial of degree eight in $\rho^{2}$ and in $z^{2}$ [20]. Depending on the values of the robot geometric parameters, $0,2,4,6$, or 8 cusps will appear in these curves [19]. Since the number of input variables is two, these curves will not contain singularities of codimension greater than 0 for generic values of the robot geometric parameters. It is certainly possible to modify these parameters to have a $3 \mathrm{R}$ robot containing a singularity of codimension 1 , a swallowtail for instance, but the robot should be deliberately designed for this purpose. Alternatively, we can consider one of the geometric parameters, say $d_{4}$, also as input variable so that the input space would be defined by $\omega_{1}=\rho, \omega_{2}=z$, and $\omega_{3}=d_{4}$. Then, by locating the singularities of codimension 1 , it is possible to find domains for the values of $d_{4}$ defining $3 \mathrm{R}$ serial robots having the same number of cusps. When crossing a boundary between two domains, the number of cusps changes as the singularity curves necessarily bifurcate as presented in the previous section.

In order to exemplify a swallowtail singularity and its bifurcation, let us set $d_{2}=1, d_{3}=4 / 5, r_{2}=1 / 2$, and $r_{3}=0$. For $d_{4}=3$, the robot has no cusps (Fig. 6a). Then, if $d_{4}$ is progressively decreased, a swallowtail singularity is obtained at $d_{4}=\frac{2}{5} \sqrt{29} \simeq 2.15$ (Fig. 6b). If $d_{4}$ is decreased further, this singularity bifurcates into two cusps. For $d_{4}=1$, these cusps are clearly visible (Fig. 6c). Using the results presented in [19], it is possible to prove that a swallowtail singularity is obtained when

$$
\left(d_{3}-1\right)^{2}\left(d_{3}^{2}-d_{4}^{2}\right)+r_{2}^{2} d_{3}^{2}=0 .
$$

In order to exemplify a beaks singularity, let us set $d_{2}=1$, 
$d_{3}=1, r_{2}=1 / 5$, and $r_{3}=9 / 10$. If $d_{4}=3 / 2$, the robot singularity locus exhibits four cusps (Fig. 6d). If $d_{4}$ is progressively decreased, two beaks singularities are encountered at $d_{4}=\frac{1}{5} \sqrt{26} \simeq 1.02$ (Fig. 6e). Then, if $d_{4}$ is further decreased, these singularities bifurcate into two pairs of cusps. For $d_{4}=1$, the resulting singularity locus has eight cusps (Fig. 6f). Again, using the results presented in [19], it can be checked that a beaks singularity is obtained for a quite simple condition:

$$
r_{2}^{2}+d_{3}^{2}-d_{4}^{2}=0
$$

In order to exemplify a lips singularity, let us set $d_{2}=1$, $d_{3}=1 / 2, r_{2}=1 / 5$, and $r_{3}=4 / 5$. For $d_{4}=1 / 4$, the singularity locus contains no cups (Fig. $6 \mathrm{~g}$ ). If $d_{4}$ is increased, a lips singularity is obtained at $d_{4} \simeq 0.33$ (Fig. 6h). This singularity also bifurcates into two cusps when $d_{4}$ is further increased. For $d_{4}=2 / 5$, these two cusps are clearly visible in the plots of the singularity locus (Fig. 6i). The algebraic characterization of the lips singularities for this robot results in a much more complicated equation, a polynomial equation of degree 12 in the geometric parameters.
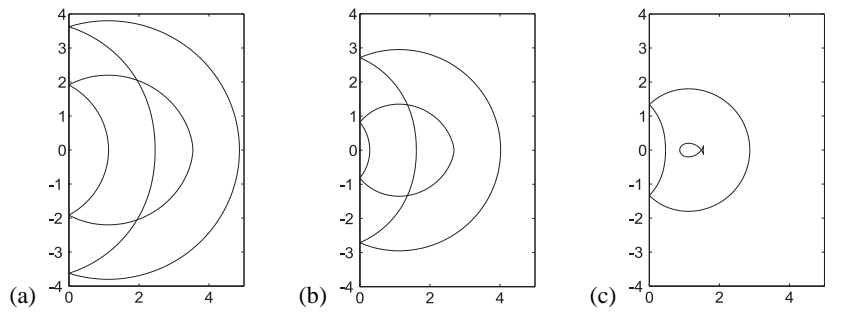

(d)
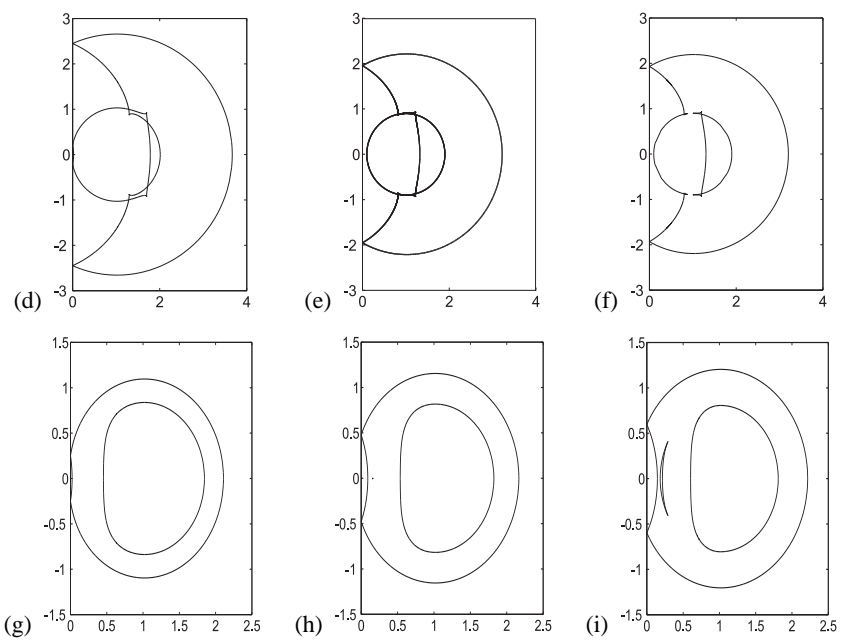

Fig. 6. Examples of the three singularities of codimension 1 and their bifurcations in the singularity locus of an orthogonal $3 \mathrm{R}$ robot. The value of the coordinates on the vertical and horizontal axes correspond to $z$ and $\rho$, respectively.

Finally, note that, when (8) and (9) are simultaneously satisfied, a gulls singularity is obtained but this is only possible if one more geometric parameter, besides $d_{4}$, is taken as variable. Solving (8) and (9) for $d_{3}$ and $d_{4}$ yields $d_{3}=1 / 2$ and $d_{4}=\sqrt{r_{2}^{2}+d_{2}^{2} / 4}$. For example, if $d_{2}=1$, $r_{2}=1 / 2$ and $r_{3}=0$, a gulls will appear when $d_{3}=1 / 2$ and $d_{4}=\sqrt{2} / 2$.

\section{Conclusions}

It has been shown how the use of Catastrophe Theory and its application to the projection of smooth surfaces is useful for the study of the structural stable properties of robot singularity loci and, in particular, to decompose these loci into domains with constant number of cups. The boundaries of these domains are determined by the so-called singularities of codimension 1 which can only be of three types. It has also been shown how these singularities bifurcate thus providing valuable information on the genesis of cusps.

\section{ACKNOWLEDGMENT}

This work has been realized during a sojourn stay of the first author at IRCCyN funded by Ecole Centrale de Nantes. The first author is also supported by the Catalan Research Commission through the Robotics Group.

\section{REFERENCES}

[1] P. Donelan, Singularities in Robot Kinematics - A Publications Database, available on-line at http://www.mcs.vuw.ac.nz/ $/$ donelan/cgi$\mathrm{bin} / \mathrm{rs} / \mathrm{main}$.

[2] G. Vegter and M. Szafraniec, "Apparent contours of implicit surfaces," Technical Report ECG-TR-124102-03, ECG, 2002.

[3] P. Wenger, "Cuspidal and non-cuspidal robot manipulator," Robotica, Vol. 25, pp.677-689, 2007.

[4] H. Bamberger, A. Wolf, M. Shoham, "Assembly Mode Changing in Parallel Mechanisms," IEEE Trans. on Robotics, Vol. 24, No. 4, pp. 765-772, 2008.

[5] R. Cipolla and P.J. Giblin, Visual Motion of Curves and Surfaces, Cambridge University Press, 2000.

[6] S. Pae and J. Ponce, "On Computing Structural Changes in Evolving Surfaces and their Appearance," International Journal of Computer Vision, Vol. 43, pp. 113-131, 2001.

[7] V.I. Arnold, Catastrophe Theory, Springer Verlag, 1992.

[8] J. Callahan, "Singularities and plane maps," The American Mathematical Monthly, Vol. 81, No. 3, pp. 211-240, 1974.

[9] H. Witney, "On singularities of mappings of Euclidean spaces I. Mappings of the plane into the plane," Annals of Mathematics, Vol. 62, No. 3, pp. 374-410, 1955.

[10] O.A. Platonova, "Singularities of projections of smooth surfaces," Russian Mathematical Surveys, Vol. 39, No. 1, pp. 177-178, 1984.

[11] C. McCrory, "Profiles of surfaces," Unpublished notes, Mathematics Institute, University of Warwick, England, 1980.

[12] Y.L. Kergosien, "La famille des projections orthogonales d'une surface et ses singularités," Comptes Rendus de l'Academie des Sciences, Vol. 11, No. 5, pp. 46-65, 1981.

[13] Y.L. Kergosien and R. Thom, "Sur les points paraboliques des surfaces," Comptes Rendus Acad. Sci. Paris, Sér. A-B 290, A705-A710, 1980.

[14] R. Uribe-Vargas, "A projective invariant for swallowtails and godrons, and global theorems on the flecnodal curve," Moscow Mathematica Journal, Vol. 6, No. 4, pp. 731-768, 2006.

[15] G. Salmon, A Treatise in Analytic Geometry of Three Dimensions, Chelsea Publ., 1927.

[16] V.S. Kulikov, "On the number of singular focal centers of the projection of an algebraic surface," Russian Mathematical Surveys, Vol. 39, No. 5, pp. 279-280, 1984.

[17] S. Plantinga and G. Vegter, "Contour generators of evolving implicit surfaces," ACM Transactions on Graphics, Vol. 25, No. 4, pp. 12431280, 2003.

[18] M.F. Roy and T. Van Effelterre, "Aspect graphs of algebraic surfaces," Proceedings of the ACM/ISSAC, pp. 135-143, 1993.

[19] P. Wenger, M. Baili, and D. Chablat, "A workspace based classification of 3R orthogonal manipulators", Proc. of the 8th Int. Symposium on Advances in Robot Kinematics, pp 219-228, 2004.

[20] F. Ranjbaran and J. Angeles, "On positioning singularities of 3revolute robotic manipulators," Proc. 12th Symp. on Engineering Applications in Mechanics, pp. 273-282, Montreal, Canada, 1994. 